\title{
Limited-Feedback Distributed Relay Selection for Random Spatial Wireless Networks
}

\author{
Hazer Inaltekin ${ }^{\dagger}$, Saman Atapattu* and Jamie Evans* \\ ${ }^{\dagger}$ School of Engineering, Macquarie University, North Ryde, NSW 2109, Australia. \\ *Department of Electrical and Electronic Engineering, University of Melbourne, Parkville, VIC 3010, Australia. \\ Email: ${ }^{\dagger}$ hazer.inaltekin@mq.edu.au; * $\{$ saman.atapattu, jse $\} @$ unimelb.edu.au
}

\begin{abstract}
This paper considers a location-based optimal relay selection scheme for a relay-assisted wireless network where available decode-and-forward relays are distributed as a homogeneous Poisson point process. To solve an optimum relay selection problem, a central entity or the source requires information pertaining to all relay locations. Since the task of feeding this information back is impractical, we investigate a threshold-based limited feedback distributed relay selection policy. We show that the total number of relays feeding back is a Poisson distributed random variable. For a given threshold-based limited feedback distributed relay selection policy, we obtain analytical expressions for the average rate and the outage probability over the fading and no-fading communication scenarios. The derived analytical expressions are verified and the performance achieved by the proposed relay selection policy is illustrated through extensive simulations. It is observed that the limited feedback distributed relay selection policy can achieve almost the same performance with the optimum relay selection policy by only utilizing location information from a few number of relays.
\end{abstract}

Index Terms-Feedback, cooperative communications, relays, Poisson point process (PPP), stochastic geometry.

\section{INTRODUCTION}

Relay-assisted wireless communications is an important means to improve the coverage and rate in next generation wireless systems. Especially, by utilizing spatial and multiuser diversity efficiently, it is envisioned that relays will be an integral part of high-frequency wireless communications in the millimeter wave bands [1]. However, the full scale of these benefits due to deployment of relays in wireless systems cannot be achieved without having a well-designed adaptive relay selection mechanism taking signal quality indicators at multiple relay locations into account [2]-[4]. It is clear that any such relay selection mechanism will require a certain level of feedback from the relay nodes while choosing the optimum relay for high-levels of communications fidelity and spectral efficiency. This is an onerous requirement in practical implementations for networks with large numbers of relays.

In this paper, we develop a low-complexity limited feedback distributed relay selection policy that requires feedback only from a few number of relays on the average. We derive some key statistical properties of the proposed relay selection policy for when relays are randomly distributed over the plane according to a homogeneous Poisson point process (HPPP). In particular, we show that the number of relays feeding their channel quality indicators back to the source node for

This work is supported in part by the Australian Research Council (ARC) through the Discovery Early Career Researcher (DECRA) Award under Grant DE160100020 and in part by the Discovery Project (DP) under Grant DP180101205. relay selection obeys to a Poisson distribution with a certain mean whose analytical form is completely characterized. The proposed feedback mechanism is fully distributed since the relay nodes solely utilize their local channel state information to decide as to whether they feed back or not. We obtain the average rate and outage probability attained by the proposed limited feedback distributed relay selection policy, and show that it is enough to multiplex only five relays over the feedback channel to achieve almost the same performance with the all-feedback scenario. From an implementation and systemdesign point of view, this presents a massive reduction in the feedback load with a negligible loss in the communications performance. An extensive simulation study is performed to illustrate and corroborate the analytical results.

Relay selection problem for randomly deployed relay networks was the subject of some previous papers, among which we briefly mention those that are most relevant to the current paper below [5]-[13]. Considering both random location and fading, a relay selection scheme which maximizes the end-toend signal-to-noise ratio (SNR) was proposed and analyzed in [5]. Another relay selection mechanism choosing the relay with the best SNR to the destination was considered in [6], and the outage probability was analyzed for different selection combining schemes. In [7], a set of relays that can successfully decode the source's message was first identified, and then the relay in this set having the minimum path loss to the destination was selected for communications. This requires the first hop location knowledge and channel state information (CSI) as well as the second hop location knowledge. Similarly, a quality-of-service (QoS) region satisfying the target outage probability was considered in [8].

In [9], the relay having the best channel to the destination was selected to forward the messages when both location knowledge and CSI are available. A similar relay selection was also considered in [10]. Based on the first hop location and channel knowledge, the work [11] considered the selection of the closest relay to the source in order to relay the messages to the destination. In [12], the optimum relay activation probability was analyzed when relays were selected based on their distances to the source node. In [13], a random relay selection policy choosing a relay in an area around the source-destination mid-point was proposed and its outage was compared with the nearest-neighbor relay selection.

All of the above previous studies assume the existence of information pertaining to all relay locations and/or wireless channels at a central entity or the source node. This operating 
assumption requires a large feedback load on the network, which does not scale well with the number of relay nodes in the network. In this paper, we focus on solving this open problem in the literature by proposing a thresholdbased limited feedback distributed relay selection policy. We obtain fundamental statistical properties for the proposed relay selection mechanism and derive its performance. As a practical design guideline, we show that having five relays on the average to feed back is enough to achieve almost the same performance with having all relays feeding back their channel quality indicators.

Notation: We use boldface, upper-case and calligraphic letters to represent vector quantities, random variables and sets, respectively. We use $\mathbb{R}, \mathbb{N}, \mathbb{C}$ and $\mathbb{R}^{2}$ to denote the real, natural and complex numbers, and the two-dimensional Euclidean space, respectively. $|x|$ denotes the absolute value of a scalar quantity $x$ (real or complex), whereas $\|\boldsymbol{x}\|$ is used to measure the canonical Euclidean norm of a vector quantity $\boldsymbol{x}$. Expected value of a random variable $X$ is denoted by $\mathrm{E}[X]$.

\section{SYSTEM MODEL}

\section{A. Network Model}

We consider a relay-assissted spatial wireless network in $\mathbb{R}^{2}$. The network contains a source-destination pair having arbitrary locations $\boldsymbol{x}_{\mathrm{s}} \in \mathbb{R}^{2}$ (source node) and $\boldsymbol{x}_{\mathrm{d}} \in \mathbb{R}^{2}$ (destination node). We will assume that $\boldsymbol{x}_{\mathrm{s}}=(-d, 0)^{\top}$ and $\boldsymbol{x}_{\mathrm{d}}=(d, 0)^{\top}$ without loss of generality. The locations of potential half-duplex (HD) decode-and-forward (DF) relay nodes in the network are given by an HPPP $\Phi=\left\{\boldsymbol{X}_{1}, \boldsymbol{X}_{2}, \ldots\right\}$ having intensity $\lambda>0$, where $\boldsymbol{X}_{i} \in \mathbb{R}^{2}$ represents the $i$ th relay location for $i \in \mathbb{N}$. When we focus on a particular realization of $\Phi$, we use $\varphi=\left\{\boldsymbol{x}_{1}, \boldsymbol{x}_{2}, \ldots\right\}$ to represent relay locations.

We will assume that the direct link between source and destination nodes is not available due to severe shadowing by an object in the environment. We will also assume that random fading coefficients change at a much faster time-scale than the network node locations, which is usually the case in typical wireless communication scenarios [14], [15]. In such cases, it is an onerous task, if not practical due to the triggered excessive relay switching rate, for the source node to obtain CSI for all source-to-relay and relay-to-destination channels, and establish a connection to another relay node for handing over the data traffic each time fading coefficients change. Hence, we will focus on relay locations as critical channel quality indicators to determine the achievable data rates between source and destination for relay-assisted wireless communications.

\section{B. Optimal Relay Selection Policy}

Our relay selection criterion will be based on the relay locations. This is also the optimum approach when only location information but not the full CSI is available at the source node. In this case, the optimum relay selection problem is equivalent to solving

$$
\begin{array}{ll}
\underset{\boldsymbol{x} \in \mathbb{R}^{2}}{\operatorname{minimize}} & \widehat{s}(\boldsymbol{x}) \\
\text { subject to } & \boldsymbol{x} \in \varphi
\end{array}
$$

for each $\varphi \in \Sigma$, where $\Sigma$ is the collection of all countable locally finite subsets of $\mathbb{R}^{2}$ and $\widehat{s}(\boldsymbol{x})=$ $\max \left\{\left\|\boldsymbol{x}_{\mathrm{s}}-\boldsymbol{x}\right\|,\left\|\boldsymbol{x}-\boldsymbol{x}_{\mathrm{d}}\right\|\right\}[16]$. The optimum relay selection policy, which we denote by $\mathcal{P}_{\text {opt }}$, is the one that solves (1) for all $\varphi \in \Sigma$. It is shown in [16] that the outage probability $P_{\text {out }}\left(\mathcal{P}_{\text {opt }}\right)$ and the average rate $\mathrm{R}_{\text {ave }}\left(\mathcal{P}_{\text {opt }}\right)$, respectively, satisfy

$$
\mathrm{P}_{\text {out }}\left(\mathcal{P}_{\text {opt }}\right)=\inf _{\mathcal{P} \in \Xi} \mathrm{P}_{\text {out }}(\mathcal{P}) ; \mathrm{R}_{\text {ave }}\left(\mathcal{P}_{\text {opt }}\right)=\sup _{\mathcal{P} \in \Xi} \mathrm{R}_{\text {ave }}(\mathcal{P}),
$$

where $\Xi$ is the set of all feasible relay selection policies, $\mathrm{P}_{\text {out }}(\mathcal{P})$ is equal to $\mathrm{P}_{\text {out }}(\mathcal{P})=\operatorname{Pr}\left\{\mathrm{R}_{\Phi}(\mathcal{P}) \leq \rho\right\}, \mathrm{R}_{\text {ave }}(\mathcal{P})$ is equal to $\mathrm{R}_{\text {ave }}(\mathcal{P})=E\left[\mathrm{R}_{\Phi}(\mathcal{P})\right]$ and $\mathrm{R}_{\Phi}(\mathcal{P})$, which is the ergodic data rate from source to destination averaged over only the fading process and can be given according to (3) at the top of the next page. When we write $\boldsymbol{X}_{\mathcal{P}}$, we refer to the location of the relay selected by $\mathcal{P}$.

\section{Distributed Relay Selection With Limited FEEDBACK}

The solution of (1) assumes a centralized operation in which information pertaining to all relay locations is available at the source node (or at a central entity). Even though this only requires relay locations as the CSI, which change at a much slower time-scale than fading, the task of feeding this information back to the source node is still onerous, and hence impeding practical implementations.

To regulate the feedback load in the network, we will use simple but practical threshold feedback policies. In particular, it is well-known that this class of feedback polices possesses certain optimality properties to maximize data rates [17]. In this paper, we will use them to control the number of relay nodes feeding their channel states back to the source node as a measure of the total feedback load in the network. More explicitly, for any given threshold value $T \geq 0$, we will say that a relay node located at $\boldsymbol{X} \in \Phi$ and operating according to a threshold-based limited feedback distributed relay selection policy with threshold value $T$ will feed its channel quality indicator $\widehat{s}(\boldsymbol{X})$ back to the source node if and only if $\widehat{s}(\boldsymbol{X}) \leq$ $T$. Hence, the total number of relays feeding back is given by

$$
N_{\mathrm{FB}}=\sum_{\boldsymbol{X} \in \Phi} 1_{\{\widehat{s}(\boldsymbol{X}) \leq T\}}
$$

where $1_{\{\cdot\}}$ is the indicator. The average number of relays feeding back is then equal to

$$
\mu(T)=\mathrm{E}\left[N_{\mathrm{FB}}\right] .
$$

The next theorem characterizes the distribution of $N_{\mathrm{FB}}$ and the functional form of its average value.

Theorem 1: For any given threshold value $T \geq 0, N_{\mathrm{FB}}$ is a Poisson distributed random variable whose mean $\mu(T)$ is given according to

$$
\mu(T)=\lambda T^{2}\left(\pi-\frac{2 d \sqrt{T^{2}-d^{2}}}{T^{2}}-2 \arctan \left(\frac{d}{\sqrt{T^{2}-d^{2}}}\right)\right)
$$




$$
\mathrm{R}_{\Phi}(\mathcal{P})=\frac{1}{2} \min \left\{\mathrm{E}\left[\log _{2}\left(1+\mathrm{SNR}\left|H_{\mathrm{s}, \mathrm{r}}\right|^{2} G\left(\left\|\boldsymbol{x}_{\mathrm{s}}-\boldsymbol{X}_{\mathcal{P}}\right\|\right)\right) \mid \Phi\right], \mathrm{E}\left[\log _{2}\left(1+\mathrm{SNR}\left|H_{\mathrm{r}, \mathrm{d}}\right|^{2} G\left(\left\|\boldsymbol{X}_{\mathcal{P}}-\boldsymbol{x}_{\mathrm{d}}\right\|\right)\right) \mid \Phi\right]\right\}
$$

for $T \geq d$, and $\mu(T)=0$ for $T<d$.

Proof: See Appendix A.

Using (6), it can easily be seen that $\mu(d)=0$ and $\mu(T)$ is a continuous function of $T$. Further, by using (4) and (5), it can also be seen that $\mu(T)$ is a monotone increasing function of $T$ with limit $\lim _{T \rightarrow \infty} \mu(T)=\infty$. Hence, for any given feedback load $\mu_{0} \geq 0$, we are guaranteed to find a threshold value $T_{0}$ such that $\mu\left(T_{0}\right)=\mu_{0}$ by intermediate value theorem. From a network design perspective, this observation shows that the class of threshold-based distributed relay selection policies is rich enough to satisfy any given feedback load constraint on the network by properly allocating a common threshold value to all relay nodes and allowing them to operate autonomously while giving their feedback decisions.

In Fig. 1, we plot the simulated distributions of $N_{\mathrm{FB}}$ for $\lambda=0.5$ and $\lambda=1$, and compare them with the Poisson distribution having mean $\mu(T)$. As predicted by Theorem 1 , simulated and theoretical distributions match each other perfectly. In particular, $\operatorname{Pr}\left\{N_{\mathrm{FB}} \geq 1\right\}$ is an important performance indicator for relay-assisted spatial wireless networks with distributed relay selection and limited feedback. The relay with optimum location is always among the relays feeding their channel quality indicators back to the source node if $N_{\mathrm{FB}} \geq 1$. Hence, with probability $\operatorname{Pr}\left\{N_{\mathrm{FB}} \geq 1\right\}$, there is no loss of optimality arising from implementing a threshold-based limited feedback distributed relay selection mechanism. Based on Theorem 1, this probability is equal to $\operatorname{Pr}\left\{N_{\mathrm{FB}} \geq 1\right\}=1-\mathrm{e}^{-\mu(T)}$. This shows that the performance loss due to having a threshold-based limited feedback distributed relay selection mechanism diminishes exponentially fast as a function of the feedback load in the network. Numerically, we have $\operatorname{Pr}\left\{N_{\mathrm{FB}} \geq 1\right\} \leq 0.99$ whenever $\mu(T) \geq 5$. As a result, for a given relay intensity and source-destination separation, choosing the threshold value such that $\mu(T)=5$ implies almost a negligible performance loss, whilst providing a massive reduction in the total feedback load required to achieve $R_{\text {ave }}\left(\mathcal{P}_{\text {opt }}\right)$ and $\mathrm{P}_{\text {out }}\left(\mathcal{P}_{\text {opt }}\right)$.

We explicitly obtain the average rate and outage probability achieved by a threshold-based limited feedback distributed relay selection policy $\mathcal{P}_{\mathrm{FB}}$ for the power-law path-loss model $\frac{1}{x^{\alpha}}$ below. With a slight abuse of notation, we will denote the average rate and outage probability by $\mathrm{R}_{\text {ave }}\left(\mathcal{P}_{\mathrm{FB}}, T\right)$ and $\mathrm{P}_{\text {out }}\left(\mathcal{P}_{\mathrm{FB}}, T\right)$, respectively, to indicate their dependency on the threshold level $T$. If $N_{\mathrm{FB}}=0$, there is no relay feeding back to the source, and we assume that the source node does not transmit any data due to lack of CSI in this case. Since $N_{\mathrm{FB}}=0$ for $T<d$ (i.e., no relay feeds back for this range of $T$ ), we will only analyze the case $T \geq d$ below. Under these operating conditions, the following theorem provides the analytical expressions for the average rate achieved by $\mathcal{P}_{\mathrm{FB}}$.

Theorem 2: For a given threshold-based limited feedback distributed relay selection policy $\mathcal{P}_{\mathrm{FB}}$ having a threshold value $T \geq d$, the average rate $\mathrm{R}_{\text {ave }}\left(\mathcal{P}_{\mathrm{FB}}, T\right)$ is equal to

$$
\mathrm{R}_{\mathrm{ave}}\left(\mathcal{P}_{\mathrm{FB}}, T\right)=\left\{\begin{array}{c}
\frac{1}{2} \int_{\frac{\mathrm{SNR}}{T^{\alpha}}}^{\frac{\mathrm{SNR}}{\mathrm{N}^{\alpha}}} \log _{2}(1+s) f_{S_{\mathrm{opt}}}(s) d s \\
\frac{1}{2 \ln 2} \int_{\frac{\mathrm{SNR}}{T^{\alpha}}}^{\frac{5 N R}{d \alpha}} \mathrm{e}^{\frac{1}{s}} \mathrm{E}_{1}\left(\frac{1}{s}\right) f_{S_{\mathrm{opt}}}(s) d s \\
\text { if Rayleigh fading }
\end{array},\right.
$$

where $\mathrm{E}_{1}(x)$ is the exponential integral defined as $E_{1}(x)=$ $\int_{1}^{\infty} \frac{\mathrm{e}^{-t x}}{t} d t$ for $x>0$ and $f_{S_{\mathrm{opt}}}(s)$ is given in [16] as

$$
\begin{aligned}
f_{S_{\mathrm{opt}}}(s)= & \frac{4 \lambda}{\alpha s}\left(\frac{\mathrm{SNR}}{s}\right)^{\frac{2}{\alpha}} \operatorname{arcsec}\left(\frac{1}{d}\left(\frac{\mathrm{SNR}}{s}\right)^{\frac{1}{\alpha}}\right) \\
& \mathrm{e}^{2 \lambda\left(d \sqrt{\left(\frac{\mathrm{SNR}}{s}\right)^{\frac{2}{\alpha}}-d^{2}}-\left(\frac{\mathrm{SNR}}{s}\right)^{\frac{2}{\alpha}} \operatorname{arcsec}\left(\frac{1}{d}\left(\frac{\mathrm{SNR}}{s}\right)^{\frac{1}{\alpha}}\right)\right)}
\end{aligned}
$$

for $0<s<\frac{\mathrm{SNR}}{d^{\alpha}}$, and $f_{S_{\mathrm{opt}}}(s)=0$ for otherwise.

Proof: The proof follows from the equivalence of events $\left\{N_{\mathrm{FB}} \geq 1\right\}$ and $\left\{\Gamma_{\mathrm{opt}} \leq T\right\}$, where $\Gamma_{\mathrm{opt}}=\widehat{s}\left(\boldsymbol{X}_{\mathrm{opt}}\right)$ and $\boldsymbol{X}_{\text {opt }}$ is the location of the relay selected by $\mathcal{P}_{\text {opt }}$. We omit the details due to space limitations.

In the next theorem, we provide similar expressions for the outage probability $\mathrm{P}_{\text {out }}\left(\mathcal{P}_{\mathrm{FB}}, T\right)$ achieved by $\mathcal{P}_{\mathrm{FB}}$.

Theorem 3: For a given threshold-based limited feedback distributed relay selection policy $\mathcal{P}_{\mathrm{FB}}$ having a threshold value $T \geq d$, the outage probability $\mathrm{P}_{\text {out }}\left(\mathcal{P}_{\mathrm{FB}}, T\right)$ is equal to

$$
\mathrm{P}_{\text {out }}\left(\mathcal{P}_{\mathrm{FB}}, T\right)= \begin{cases}\mathrm{e}^{-\mu(T)} & \text { if } \rho \leq \mathrm{R}_{1} \\ F_{S_{\text {opt }}}\left(2^{2 \rho}-1\right) & \text { if } \mathrm{R}_{1}<\rho<\mathrm{R}_{2} \\ 1 & \text { if } \rho \geq \mathrm{R}_{2}\end{cases}
$$

when there is no fading, where $\mathrm{R}_{1}=\frac{1}{2} \log _{2}\left(1+\frac{\mathrm{SNR}}{T^{\alpha}}\right), \mathrm{R}_{2}=$ $\frac{1}{2} \log _{2}\left(1+\frac{\mathrm{SNR}}{d^{\alpha}}\right)$ and $F_{S_{\mathrm{opt}}}$ is given in [16] as

$$
F_{S_{\mathrm{opt}}}(s)=\left\{\begin{array}{ll}
0 & \text { if } s \leq 0 \\
\mathrm{e}^{2 \lambda\left(d \sqrt{\left(\frac{\mathrm{SNR}}{s}\right)^{\frac{2}{\alpha}}-d^{2}}-\left(\frac{\mathrm{SNR}}{s}\right)^{\frac{2}{\alpha}} \operatorname{arcsec}\left(\frac{1}{d}\left(\frac{\mathrm{SNR}}{s}\right)^{\frac{1}{\alpha}}\right)\right)} \\
1 & \text { if } 0<s<\frac{\mathrm{SNR}}{d^{\alpha}}
\end{array} .\right.
$$

On the other hand, $\mathrm{P}_{\text {out }}\left(\mathcal{P}_{\mathrm{FB}}, T\right)$ is equal to

$$
\mathrm{P}_{\text {out }}\left(\mathcal{P}_{\mathrm{FB}}, T\right)= \begin{cases}\mathrm{e}^{-\mu(T)} & \text { if } s^{\star} \leq \frac{\mathrm{SNR}}{T^{\alpha}} \\ F_{S_{\mathrm{opt}}}\left(s^{\star}\right) & \text { if } \frac{\mathrm{SNR}}{T^{\alpha}}<s^{\star}<\frac{\mathrm{SNR}}{d^{\alpha}} \\ 1 & \text { if } s^{\star} \geq \frac{\mathrm{SNR}}{d^{\alpha}}\end{cases}
$$

for Rayleigh distributed fading, where $s^{\star}$ is the unique solution of the equation $\frac{1}{2 \ln 2} \mathrm{e}^{1 / s} \mathrm{E}_{1}(1 / s)=\rho$.

Proof: We give the proof only for the fading case due to space limitations. In this case, we can write the outage event as the union of two disjoint events according to

$$
\begin{aligned}
& \left\{\mathrm{R}_{\Phi}\left(\mathcal{P}_{\mathrm{FB}}, T\right) \leq \rho\right\} \\
& =\left\{\Gamma_{\mathrm{opt}}>T\right\} \bigcup\left(\left\{\Gamma_{\mathrm{opt}} \leq T\right\} \bigcap\left\{\frac{1}{2 \ln 2} f\left(\frac{\Gamma_{\mathrm{opt}}^{\alpha}}{\mathrm{SNR}}\right)\right\} \leq \rho\right),
\end{aligned}
$$



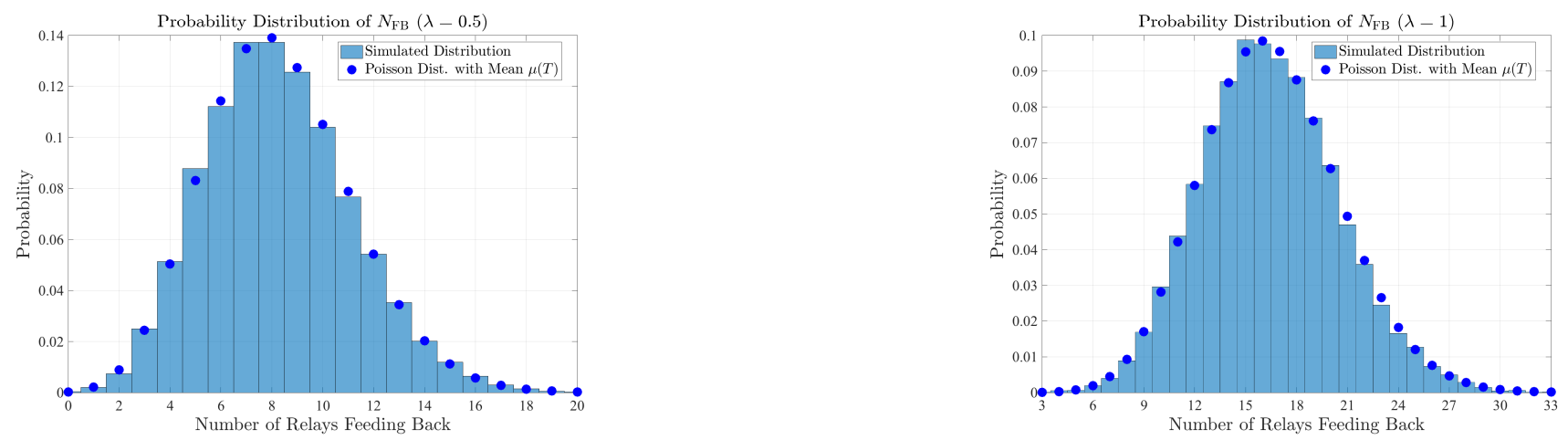

Fig. 1: Probability distribution of the number of relays feeding back for $T=3$ and $d=1 .(\lambda=0.5$ for the left-hand side figure and $\lambda=1$ for the right-hand side figure.)

where $f(x)$ is defined to be $f(x) \triangleq \mathrm{e}^{x} \mathrm{E}_{1}(x)$ for $x>0$. Hence, we can write

$$
\begin{aligned}
& \mathrm{P}_{\text {out }}\left(\mathcal{P}_{\mathrm{FB}}, T\right) \\
& =\mathrm{e}^{-\mu(T)}+\operatorname{Pr}\left(\left\{\Gamma_{\mathrm{opt}} \leq T\right\} \bigcap\left\{\frac{1}{2 \ln 2} f\left(\frac{\Gamma_{\mathrm{opt}}^{\alpha}}{\mathrm{SNR}}\right)\right\} \leq \rho\right) .
\end{aligned}
$$

It can be shown that $f(x)$ is a continuous and strictly decreasing function of $x$ with limiting values $\lim _{x \rightarrow 0} f(x)=\infty$ and $\lim _{x \rightarrow \infty} f(x)=0$. Hence, defining $\gamma^{\star}$ as the unique solution of the equation $\frac{1}{2 \ln 2} f\left(\frac{\gamma^{\star \alpha}}{\mathrm{SNR}}\right)=\rho$, analyzing three cases $\gamma^{\star} \leq d, d<\gamma^{\star}<T$ and $\gamma^{\star} \geq T$ separately, and

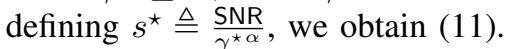

Several important remarks are in order about Theorem 3 characterizing the outage probability achievable with a threshold-based limited feedback distributed relay selection policy. In particular, we observe two regimes emerging in Theorem 3 when we vary the target rate for both with and without fading. When $\rho \leq \frac{1}{2} \log _{2}\left(1+\frac{\mathrm{SNR}}{T^{\alpha}}\right)$ without fading or $s^{\star} \leq \frac{\text { SNR }}{T^{\alpha}}$ with fading where $s^{\star}$ is the solution for $\frac{1}{2 \ln 2} \mathrm{e}^{1 / s} \mathrm{E}_{1}(1 / s)=\rho$, the outage probability is equal to $\mathrm{P}_{\text {out }}\left(\mathcal{P}_{\mathrm{FB}}, T\right)=\mathrm{e}^{-\mu(T)}$. This is the feedback-limited regime in which $\mathrm{P}_{\text {out }}\left(\mathcal{P}_{\mathrm{FB}}, T\right)$ is the same for both no-fading and fading cases, depends only the average number of relays feeding back (which in turn depends on $T, \lambda$ and $d$ ), and is independent of the target rate and fading behaviour. In this regime, the threshold value is set so small that we are guaranteed to achieve the target rate whenever there is at least one relay feeding its channel quality indicator back to the source node. We recall that the smaller $T$ is, the relays feeding back have the better channel gains and achieve higher rates.

The second regime is the rate-limited regime that emerges when $\frac{1}{2} \log _{2}\left(1+\frac{\mathrm{SNR}}{T^{\alpha}}\right)<\rho<\frac{1}{2} \log _{2}\left(1+\frac{\mathrm{SNR}}{d^{\alpha}}\right)$ for the no-fading case and when $\frac{\mathrm{SNR}}{T^{\alpha}}<s^{\star}<\frac{\mathrm{SNR}}{d^{\alpha}}$ for the fading case. In this regime, the outage probability is equal to $\mathrm{P}_{\text {out }}\left(\mathcal{P}_{\mathrm{FB}}, T\right)=F_{S_{\mathrm{opt}}}\left(2^{2 \rho}-1\right)$ for the no-fading case and $\mathrm{P}_{\text {out }}\left(\mathcal{P}_{\mathrm{FB}}, T\right)=F_{S_{\text {opt }}}\left(s^{\star}\right)$ for the fading case, and hence $\mathrm{P}_{\text {out }}\left(\mathcal{P}_{\mathrm{FB}}, T\right)$ is a function of $\rho$, the same for the allfeedback and limited feedback cases, and independent of $T$. $\mathrm{P}_{\text {out }}\left(\mathcal{P}_{\mathrm{FB}}, T\right)$ also depends on the fading behaviour since $s^{\star}$ is not necessarily the same with $2^{2 \rho}-1$. In this regime, the threshold value is set so big that we are guaranteed to have at least one relay feeding its channel quality indicator back to the source node whenever any relay achieves the target rate.

\section{Numerical Results}

In this section, we present our numerical results illustrating the analytical average rate and outage probability curves in Theorems 2 and 3. In Fig. 2, we plot the average rate $\mathrm{R}_{\text {ave }}\left(\mathcal{P}_{\mathrm{FB}}, T\right)$ achieved by the threshold-based limited feedback distributed relay selection policy $\mathcal{P}_{\mathrm{FB}}$ as a function of $\lambda$ for various values of $T \geq d$, where we set $d=1, \mathrm{SNR}=5 \mathrm{~dB}$ and $\alpha=4$. For small values of $T$, there is a large gap between the average rates achieved by $\mathcal{P}_{\mathrm{FB}}$ and all-feedback policies. This is due to the fact that $\operatorname{Pr}\left\{N_{\mathrm{FB}}=0\right\}=\mathrm{e}^{-\mu(T)}$ is large for small values of $T$, and hence the source node cannot receive any CSI from relays to choose one with high probability. More specifically, for the considered range of $\lambda \in[0.01,4]$ in Fig. $2, \mu(T)$ ranges from 0.0123 to 0.4934 for $T=1.1$, which indicates that the source node is without any CSI more than $60 \%$ of time even for the most crowded relay network scenario considered in this figure.

A similar behaviour with a decreased rate gap between the limited feedback and all-feedback cases continues to hold for $T=1.25$. In this case, the source node can access to CSI more than $87 \%$ of time when $\lambda=4$. For $T=1.5, \mu(T)$ is approximately equal to $3.1,4.65$ and 6.2 for $\lambda=2,3$ and 4 , respectively. As observed in Fig. 2, the rate gap between the limited feedback and all-feedback cases becomes very small after $\lambda \geq 2$ for $T=1.5$, which implies a significant reduction in the feedback load without sacrificing from the achievable data rates. Similar observations but with a smaller rate gap continues to hold for $T=2$. Based on our observations in Fig. 2 and earlier explanations after Theorem 1, as a practical network design rule of thumb, we can say that setting $T$ such that $\mu(T)=5$ is enough to achieve the same average rate attained by the all-feedback policy with almost negligible performance loss.

In Fig. 3, we plot the outage probability curves achieved by $\mathcal{P}_{\mathrm{FB}}$ for both as a function of $\lambda$ and $\rho$. As in Fig. 2, simulation and analytical results perfectly match each other. Feedbacklimited and rate-limited regimes discussed after Theorem 3 for outage probability are also apparent in this figure. While drawing $\mathrm{P}_{\text {out }}\left(\mathcal{P}_{\mathrm{FB}}, T\right)$ in the top figures, we set $\rho=0.3$. 

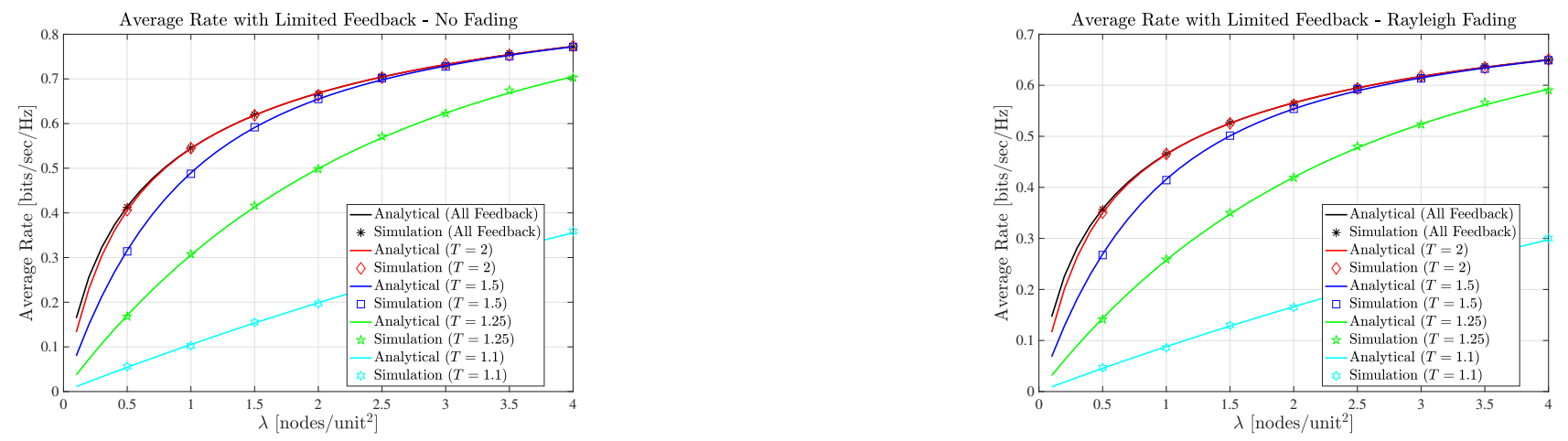

Fig. 2: Average rate achieved by the threshold-based limited feedback distributed relay selection policy for various values of the threshold level, $d=1$, SNR $=5 \mathrm{~dB}$ and $\alpha=4$. (No fading is assumed for the left-hand side figure and Rayleigh fading with unit power is assumed for the right-hand side figure.)
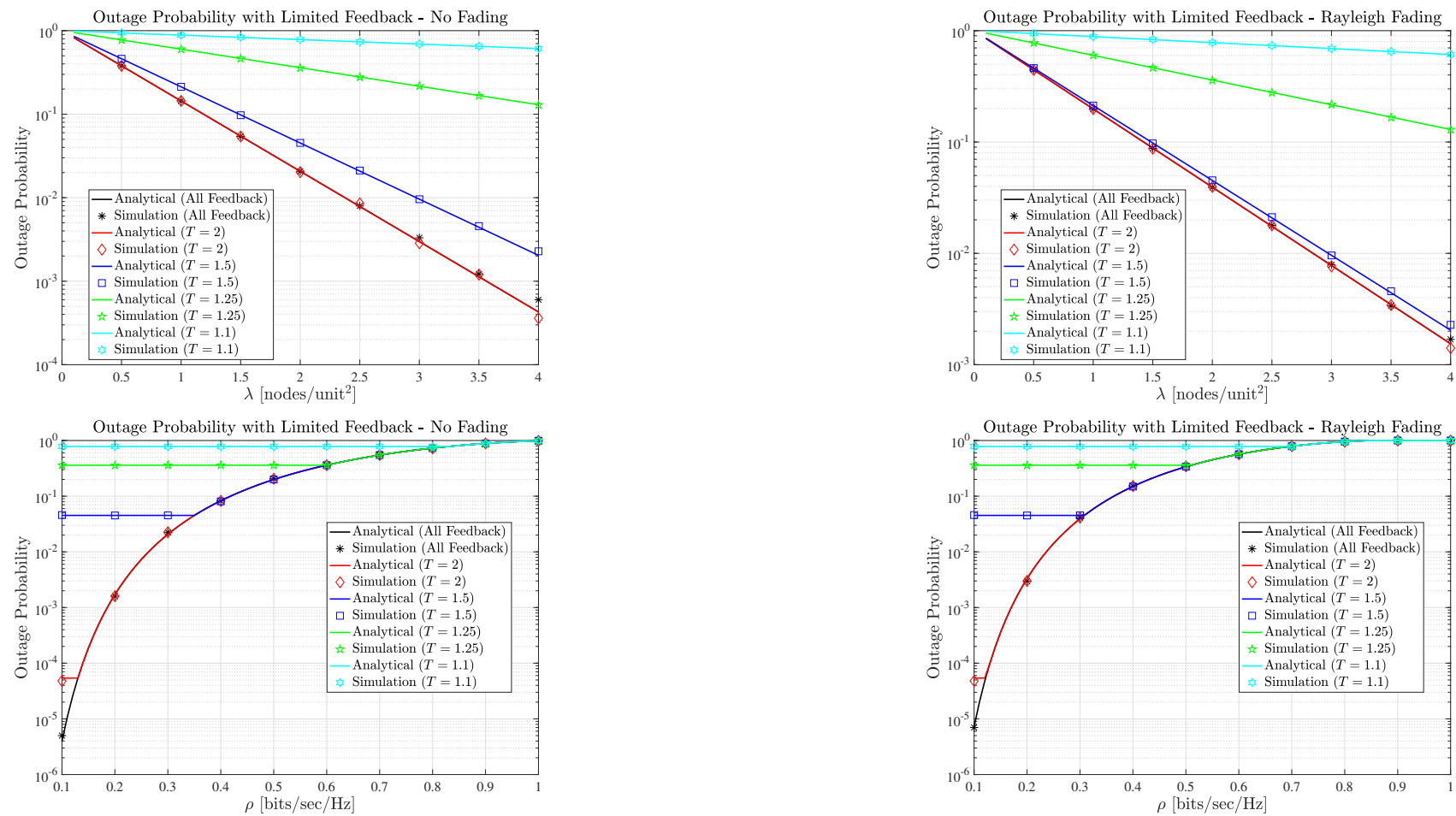

Fig. 3: Outage probability achieved by the threshold-based limited feedback distributed relay selection policy for various values of the threshold level, $d=1, \mathrm{SNR}=5 \mathrm{~dB}$ and $\alpha=4$. The outage probability curves in the top figures are as a function of $\lambda$ with $\rho$ fixed at 0.3 . The bottom ones are as a function of $\rho$ with $\lambda$ fixed at 2. (No fading is assumed for the left-hand side figures and Rayleigh fading with unit power is assumed for the right-hand side figures.)

For this value of $\rho, s^{\star}=0.6022$. Hence, we are in the feedback-limited regime for $T=1.1,1.25$ and 1.5 , and we observe exactly the same outage probabilities for both nofading and fading cases. On the other hand, we are in the ratelimited regime for $T=2$, and the outage probabilities become the same for both limited feedback and all-feedback relay selections, i.e., see the red and black curves in the top figures. As a function of $\rho$, the feedback-limited regime is manifested through the flat portion $\mathrm{P}_{\text {out }}\left(\mathcal{P}_{\mathrm{FB}}, T\right)$ in the bottom figures. In particular, when drawn as a function of $\rho, \mathrm{P}_{\text {out }}\left(\mathcal{P}_{\mathrm{FB}}, T\right)$ stays constant until a critical target rate, which is the feedbacklimited regime. In this regime, the outage probability depends only on the average number of relays feeding back, i.e., $\mathrm{P}_{\text {out }}\left(\mathcal{P}_{\mathrm{FB}}, T\right)=\mathrm{e}^{-\mu(T)}$. On the other hand, after a critical target rate value, outage probability curves coincide with each other and move together as a function of $\rho$ for both limited feedback and all-feedback scenarios. This is the rate-limited case, and the outage probability is independent of whether we employ a threshold-based limited feedback relay selection policy or not.

\section{CONCLUSIONS}

In this paper, we have considered a relay-assisted wireless network with a single source-destination pair and spatially deployed decode-and-forward relays. For a threshold-based 
limited feedback distributed relay selection policy, we have shown that the total number of relays feeding back is a Poisson distributed random variable. We have characterized the average value for this Poisson distribution analytically, and obtained the analytical expressions for the average rate and outage probability achieved by the proposed threshold-based limited feedback distributed relay selection policy for both the nofading and fading communications scenarios. We have derived useful and practical design rules for the limited feedback relay selection, which indicates that setting the threshold value to have five relay nodes feeding back on the average is enough to achieve the same communications performance attained by the all-feedback policy with almost negligible performance loss. The performance loss becomes insignificant especially when the relay intensity increases.

\section{APPENDIX A \\ PROOF OF THEOREM 1}

To prove this theorem, we first focus on the relay nodes located inside the disc $\mathcal{B}(\mathbf{0}, \tau)$, which is centered around the origin 0 and having radius $\tau$. Let $\mu(T, \tau)$ be the average number of relays located in $\mathcal{B}(\mathbf{0}, \tau)$ and feeding their channel quality indicators back to the source node. $\mu(T, \tau)$ is equal to

$$
\mu(T, \tau)=\mathrm{E}\left[\sum_{\boldsymbol{X} \in \Phi \cap \mathcal{B}(\mathbf{0}, \tau)} 1_{\{\widehat{s}(\boldsymbol{X}) \leq T\}}\right]
$$

Using the monotone convergence theorem, it can be seen that $\mu(T)=\lim _{\tau \rightarrow \infty} \mu(T, \tau)$. Let $N$ be the number of relays in $\Phi \cap \mathcal{B}(\mathbf{0}, \tau)$. Given the event $\{N=n\}$, all the relays are independently and uniformly distributed over $\mathcal{B}(\mathbf{0}, \tau)$, and hence $\mathrm{E}\left[\sum_{\boldsymbol{X} \in \Phi \cap \mathcal{B}(\mathbf{0}, \tau)} 1_{\{\widehat{s}(\boldsymbol{X}) \leq T\}} \mid N=n\right]=n \operatorname{Pr}\{\widehat{s}(\boldsymbol{U} \leq T)\}$, where $\boldsymbol{U}$ is a generic random variable uniformly distributed over $\mathcal{B}(\mathbf{0}, \tau)$. Using this observation, we can write $\mu(T, \tau)$ as

$$
\mu(T, \tau)=\lambda \pi \tau^{2} \operatorname{Pr}\{\widehat{s}(\boldsymbol{U}) \leq T\} .
$$

Now, we are interested in calculating the probability $\operatorname{Pr}\{\widehat{s}(\boldsymbol{U}) \leq T\}$, which is given by the following lemma.

Lemma 4: Let $\boldsymbol{U}$ be a random variable uniformly distributed over $\mathcal{B}(\mathbf{0}, \tau)$. Then, $\operatorname{Pr}\{\widehat{s}(\boldsymbol{U})>T\}$ for $T \geq 0$ is given by

$$
\operatorname{Pr}\{\widehat{s}(\boldsymbol{U})>T\}=\left\{\begin{array}{l}
1 \text { if } T<d \\
\frac{\tau^{2}-T^{2}}{\tau^{2}}+\rho_{\tau}(T, d) \text { if } d \leq T \leq \sqrt{\tau^{2}+d^{2}} \\
\frac{2 b^{*}\left(\tau^{2}-T^{2}\right)}{\pi \tau^{2}}-\frac{d^{2} \sin \left(2 b^{*}\right)}{\pi \tau^{2}}+\rho_{\tau}\left(T, d \sin \left(b^{*}\right)\right) \\
\text { if } \sqrt{\tau^{2}+d^{2}} \leq T \leq \tau+d \\
0 \text { if } T>\tau+\bar{d}
\end{array}\right.
$$

where $b^{*}=\arccos \left(\frac{T^{2}-\tau^{2}-d^{2}}{2 d \tau}\right)$, and

$$
\rho_{\tau}(T, d) \triangleq \frac{2 d \sqrt{T^{2}-d^{2}}}{\pi \tau^{2}}+\frac{2 T^{2}}{\pi \tau^{2}} \arctan \left(\frac{d}{\sqrt{T^{2}-d^{2}}}\right) .
$$

Proof: The proof is omitted due to space limitations.

We will use Lemma 4 to conclude the proof, and it is enough to focus only on the case where $d \leq T \leq \sqrt{d^{2}+\tau^{2}}$. In particular, it can be seen by using this lemma that $\operatorname{Pr}\{\widehat{s}(\boldsymbol{U} \leq T)\}=0$ for all values of $T$ smaller than $d$.
Therefore, $\mu(T)=\lim _{\tau \rightarrow \infty} \mu(T, \tau)=0$ for $T<d$. For other cases of this lemma where $T \geq \sqrt{d^{2}+\tau^{2}}$, the threshold value grows without any bound when $\tau$ tends to infinity, which is equivalent to the all-feedback case investigated in [16].

For $d \leq T \leq \sqrt{d^{2}+\tau^{2}}$, we have $\operatorname{Pr}\{\widehat{s}(\boldsymbol{U} \leq T)\}=$ $\frac{T^{2}}{\tau^{2}}-\rho_{\tau}(T, d)$ by using Lemma 4. As a result, $\mu(T, \tau)=$ $\lambda \pi T^{2}-\lambda \pi \tau^{2} \rho_{\tau}(T, d)$. Taking the limit as $\tau$ tends to $\infty$, we obtain (6). To obtain the distribution of $N_{\mathrm{FB}}$, we first observe that the sum $\sum_{\boldsymbol{X} \in \Phi \cap \mathcal{B}(\mathbf{0}, \tau)} 1_{\{\widehat{s}(\boldsymbol{X}) \leq T\}}$ has the characteristic function $\varphi_{\tau}(t)=\exp \left(\mu(\tau, T)\left(\mathrm{e}^{\jmath t}-1\right)\right)$. Since $N_{\mathrm{FB}}=\lim _{\tau \rightarrow \infty}=\sum_{\boldsymbol{X} \in \Phi \cap \mathcal{B}(\mathbf{0}, \tau)} 1_{\{\widehat{s}(\boldsymbol{X}) \leq T\}}$ almost surely, that $N_{\mathrm{FB}}$ has a Poisson distribution with mean $\mu(T)$ [18].

\section{REFERENCES}

[1] R. Pabst, B. H. Walke, D. C. Schultz, P. Herhold, H. Yanikomergolu, S. Mukherjee, H. Viswanathan, M. Lott, W. Zirwas, M. Dohler, H. Aghvami, D. D. Falconer, and G. P. Fettweis, "Relay-based deployment concepts for wireless and mobile broadband radio," IEEE Commun. Mag., vol. 42, no. 9, pp. 80-89, Sep. 2004.

[2] R. Tannious and A. Nosratinia, "Spectrally-efficient relay selection with limited feedback," IEEE J. Select. Areas Commun., vol. 26, no. 8, pp. 1419-1428, Oct. 2008

[3] S. Atapattu, P. Dharmawansa, M. Di Renzo, C. Tellambura, and J. S. Evans, "Multi-user relay selection for full-duplex radio," IEEE Trans. Commun., vol. 67, no. 2, pp. 955-972, Feb. 2019.

[4] S. Atapattu, N. Ross, Y. Jing, Y. He, and J. S. Evans, "Physical-layer security in full-duplex multi-hop multi-user wireless network with relay selection," IEEE Trans. Wireless Commun., vol. 18, no. 2, pp. 12161232, Feb. 2019.

[5] A. Behnad, A. M. Rabiei, and N. C. Beaulieu, "Performance analysis of opportunistic relaying in a poisson field of amplify-and-forward relays," IEEE Trans. Commun., vol. 61, no. 1, pp. 97-107, Jan. 2013.

[6] M. Mohammadi and H. A. S. and, "Outage probability of wireless ad hoc networks with cooperative relaying," in Proc. IEEE Global Telecommn. Conf. (GLOBECOM), Dec. 2012, pp. 4410-4416.

[7] K. Belbase, Z. Zhang, H. Jiang, and C. Tellambura, "Coverage analysis of millimeter wave decode-and-forward networks with best relay selection," IEEE Access, vol. 6, pp. 22 670-22 683, 2018.

[8] S. Cho, W. Choi, and K. Huang, "QoS provisioning relay selection in random relay networks," IEEE Trans. Veh. Technol., vol. 60, no. 6, pp. 2680-2689, Jul. 2011.

[9] A. Tukmanov, S. Boussakta, Z. Ding, and A. Jamalipour, "Outage performance analysis of imperfect-CSI-based selection cooperation in random networks," IEEE Trans. Commun., vol. 62, no. 8, pp. 27472757, Aug. 2014.

[10] Y. Zhou and W. Zhuang, "Performance analysis of cooperative communication in decentralized wireless networks with unsaturated traffic," IEEE Trans. Wireless Commun., vol. 15, no. 5, pp. 3518-3530, May 2016.

[11] H. Elkotby and M. Vu, "Uplink user-assisted relaying in cellular networks," IEEE Trans. Wireless Commun., vol. 14, no. 10, pp. 54685483, Oct. 2015.

[12] A. Altieri, L. R. Vega, P. Piantanida, and C. G. Galarza, "Analysis of a cooperative strategy for a large decentralized wireless network," IEEE/ACM Trans. Netw., vol. 22, no. 4, pp. 1039-1051, Aug. 2014.

[13] H. Elkotby and M. Vu, "Outage performance of uplink user-assisted relaying in 5G cellular networks," in Proc. IEEE Global Telecommn. Conf. (GLOBECOM), Dec. 2015.

[14] D. Tse and P. Viswanath, Fundamentals of Wireless Communication. New York, NY, USA: Cambridge University Press, 2005.

[15] A. Goldsmith, Wireless Communications. New York, NY, USA: Cambridge University Press, 2005.

[16] S. Atapattu, H. Inaltekin, and J. Evans, "Location-based optimum relay selection in random spatial networks," in Proc. IEEE International Conference on Communications (ICC'19), Shangai, China, May 2019.

[17] T. Samarasinghe, H. Inaltekin, and J. S. Evans, "Optimal selective feedback policies for opportunistic beamforming," IEEE Trans. Inf. Theory, vol. 59, no. 5, pp. 2897-2913, May 2013.

[18] P. Billingsley, Probability and Measure, 3rd ed. New York, NY, USA: John Wiley \& Sons, 1995. 